www.jmscr.igmpublication.org

Impact Factor 5.84

Index Copernicus Value: 83.27

ISSN (e)-2347-176x ISSN (p) 2455-0450

crossref DOI: _https://dx.doi.org/10.18535/jmscr/v5i4.72

\title{
Reward Pathway in Brain and Alcohol Dependence: A Co-Relation Study in Wistar Rats to Show the Effect on Brain Dopamine, Dihydrophenylacetic Acid (DOPAC) and Homovanilic Acid (HVA)
}

\author{
Authors
}

\section{Janki Malli, Nimmy Chacko, Vijayanarayana K}

Department of Pharmacology, NGSM Institute of Pharmaceutical Sciences, Paneer, Deralakatte,

Mangalore, Karnataka, India

\begin{abstract}
Humans, as well as other organisms engage in behaviors that are rewarding. The pleasurable feelings provide positive reinforcement so that the behavior is repeated. Each of the naturally rewarded behaviors is required for the survival of the species, so the reward pathway is a crucial part of the brain, as well as a key in understanding addictions. The major part of reward pathway is the ventral tegmental area (VTA). The neurons of the VTA contain the neurotransmitter dopamine which is released in the nucleus accumbens. This pathway is activated by a rewarding stimulus. Dopamine is released particularly in areas such as the nucleus accumbens and prefrontal cortex by naturally rewarding experiences such as food, drugs, and neutral stimuli that become associated with them. Dopamine is also a neurohormone released by the hypothalamus. Dopamine is commonly associated with the pleasure system of the brain, providing feelings of enjoyment and reinforcement to motivate a person to perform certain activities. Chemical or surgical interruption of this dopaminergic pathway impairs drug seeking behavior in many experimental situations. Deletion of D2 dopamine receptors in transgenic mouse strain eliminated the reward properties of morphine administration. Dopaminergic antagonist, like Quetiapine and Olanzapine were studied as new pharmacotherapy for treating alcohol dependence by analysing dopamine level in rat brain homogenate using HPLC. Analysis of dopamine level in rat brain homogenate using HPLC showed that the peak area of olanzapine and quetiapine group was $94.94 \mathrm{mV}$.s and $147.9 \mathrm{mV}$.s respectively which is less than peak area of alcohol $190.69 \mathrm{mV}$.s. This indicates that alcohol abusestimulates the reward pathway which increases the level of dopamine in ventral tegmental area of the brain which is antagonised by dopamine antagonists. Since dopamine plays an important role in alcohol dependence (positive reinforcement), D2 dopaminergic antagonists like Quetiapine and Olanzapine provides a new role in pharmacotherapy of alcohol dependence and alcohol withdrawal.
\end{abstract}

Keywords: Alcohol dependence, dopamine, reward pathway, mesolimbic dopaminergic pathway, dopamine antagonists, Quetiapine, Olanzapine.

\section{Introduction}

Humans, as well as other organisms engage in behaviors that are rewarding. The pleasurable feelings provide positive reinforcement so that the behavior is repeated. There are natural rewards such as food, water, and nurturing, as well as artificial rewards such as drugs.
Each of these naturally rewarded behaviors is required for the survival of the species, so the reward pathway is a crucial part of the brain, as well as a key in understanding addictions. The major structures are: the ventral tegmental area (VTA), the nucleus accumbens and the prefrontal cortex. The VTA is connected to both the nucleus 
accumbens and the prefrontal cortex via this pathway and it sends information tothese structures via its neurons. The neurons of the VTA contain the neurotransmitter dopamine which is released in the nucleus accumbens and in the prefrontal cortex. This pathway is activated by a rewarding stimulus.

The Discovery of the Reward Pathway- The discovery of the reward pathwaywas achieved with the help of animals such as rats. Rats were trained to press a lever for a tiny electrical jolt to certain parts of the brain. When an electrode isplaced in the nucleus accumbens, the rat keeps pressing the lever to receive thesmall electrical stimulus because it feels pleasurable. This rewarding feeling is also called positive reinforceement. It has been shown that when an electrode is placed an area around the nucleus accumbens, the rat will not press thelever for the electrical stimulus because stimulating neurons in a nearby area that does not connect with the nucleus accumbens does not activate the reward pathway. An increase release of neurotransmitter dopamine in the reward pathway after the rat receives the reward has been determined in these experiments.

\section{Different types of Reward pathway}

\section{a) Themesolimbic pathway}

The mesolimbic pathway is one of the neural pathways in the brain that links the ventral tegmentum in the midbrain to the nucleus accumbens, which is located in the striatum and is a part of the limbic system. It is one of the four major pathways where the neurotransmitter dopamine is found. The mesolimbic pathway is thought to be involved in producing pleasurable feeling, and is often associated with feelings of reward and desire. The mesolimbic pathway is one of the major pathways targeted by antipsychotic medication. Although the process is not fully understood.

\section{b) Themes cortical pathway}

The mesocortical pathway is a neural pathway that connects the ventral tegmentum to the cerebral cortex, particularly the frontal lobes. It is one of the four major dopamine pathways in the brain, and it is thought to be involved in motivation and emotional response. This pathway may be the brain system that is abnormal or functioning abnormally in psychoses, such as schizophrenia. It is thought to be associated with the negative symptoms of schizophrenia. The pathway is closely associated with the mesolimbic pathway, also known as the reward pathway.

\section{c) The Nigrostriatal pathway}

The nigrostriatal pathway is a neural pathway that connects the substantianigra with the striatum. It is one of the four major dopamine pathways in the brain, and is particularly involved in the production of movement. Loss of dopamine neurons in the substantianigra is one of the main pathological features of Parkinson's disease, leading to a marked reduction in dopamine function in this pathway. This pathway is also implicated in producing tardive dyskinesia, one of the side-effects of antipsychotic drugs.

\section{d) The tuberoinfundibular pathway}

The tuberoinfundibular pathway refers to a population of dopamine neurons in the arcuate nucleus of the mediobasal hypothalamus that project to the median eminence. Dopamine released at this site regulates the secretion of prolactin from the anterior pituitary gland. Some antipsychotic drugs block dopamine in the tuberoinfundibular pathway, which can cause hyper prolactinemia. This can cause abnormal laction, disruptions to the menstrual cycle in women, visual problems, headache and sexual dysfunction.

\section{Controversy of Mesolimbic Dopamine}

Function - There is some controversy regarding dopamine's role in the reward system. Three hypotheses have been proposed as explanations for dopamine's function in the reward system. They are:

(1) Hedonia - The hedonia hypothesis suggests that dopamine in the nucleus accumbens acts as a 'pleasure neurotransmitter'. However, not all rewards or pleasurable things involve activation of the reward system, which may suggest that the mesolimbic pathway may not be just a system that works merely off enjoyable things (hedonia). 
(2) Learning - Learning, on the other hand, deals with predictions of future reward. Studies have shown that rats that had their ventral tegmental area and nucleus accumbens destroyed do not lose their learning capabilities, but rather lack the motivation to work for a reward.

(3) Incentive salience - Incentive salience stands out as a possible role for dopamine as being released when there is a stimulus, thus making an individual work to get it. In self-administration studies, animals have been trained to give an operant response in order to obtain either a drug or mate. It has been shown that the animals will continue to perform the required task until the reward is received, or fatigue sets in.

Clinical significance of reward pathway - Since the mesolimbic pathway is shown to be associated with feelings of reward and desire, this pathway is heavily implicated in neurobiological theories of addiction, schizophrenia, and depression. Drug addiction, the loss of control over drug use or the compulsive seeking and taking of drugs despite adverse consequences are due to increased dopamine transmission in the limbic system each by different mechanisms.

\section{Role of Dopamine in Reinforcement}

Dopamine is a neurotransmitter occurring in a wide variety of animals, including both vertebrates and invertebrates. In the brain, this phenethylamine functions as a neurotransmitter, activating the five types of dopamine receptors $\mathrm{D}_{1}, \mathrm{D}_{2}, \mathrm{D}_{3}, \mathrm{D}_{4}$, and $\mathrm{D}_{5}$, and their variants. Dopamine is produced in several areas of the brain, including the substantianigra and the ventral tegmental area. Dopamine is also a neurohormone released by the hypothalamus. Its main function as a hormone is to inhibit the release of prolactin from the anterior lobe of the pituitary.

\section{Structure of dopamine}

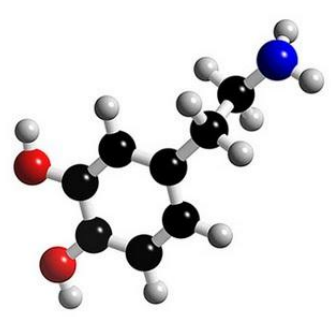

Dopamine can be supplied as a medication that acts on the sympathetic nervous system, producing effects such as increased heart rate and blood pressure. However, because dopamine cannot cross the blood-brain barrier, L-DOPA (levodopa), which is the precursor of dopamine, can be given to increase the amount of dopamine in the brains of patients with diseases such as Parkinson's disease.

Dopamine has many functions in the brain, including important roles in behavior and cognition, voluntary movement, motivation and reward, inhibition of prolactin production, sleep, mood, attention, and learning. Dopamine is commonly associated with the pleasure system of the brain, providing feelings of enjoyment and reinforcement to motivate a person to perform certain activities. Dopamine is released particularly in areas such as the nucleus accumbens and prefrontal cortex by naturally rewarding experiences such as food, drugs, and neutral stimuli that become associated with them. Recent studies indicate that aggression may also stimulate the release of dopamine in this way. This theory is often discussed in terms of drugs such as cocaine, nicotine, alcohol and amphetamines, which directly or indirectly lead to an increase of dopamine in the mesolimbic reward pathway of the brain. Cerebral dopamine depletion is the hallmark of Parkinson's disease. Other pathological states have also been associated with dopamine dysfunction, such as schizophrenia, autism, and attention deficit hyperactivity disorder in children, as well as drug abuse.

Today, alcohol is widely consumed. Like other sedative-hypnotic drugs, alcohol in low to moderate amounts relieves anxiety and fosters a feeling of well-being or even euphoria. However, alcohol is also the most commonly abused drug in the world, and a cause of vast medical and societal costs. People who continue to drink alcohol in spite of adverse medical or social consequences related directly to their alcohol consumption suffer from alcoholism, a complex disorder that appears to have genetic as well as environmental determinants. 
Olanzapine is a atypical antipsychotic resembles Clozapine in blocking multiple monoaminergic $\left(\mathrm{D}_{2}, 5-\mathrm{HT}_{2}\right)$ muscarinic and $\mathrm{H}_{1}$ receptors. Both positive and negative symptoms of schizophrenia appeared to be benefitted. Monotherapy with Olanzapine may be as effective as a combination of lithium/valproate and benzodiazepines. Olanzapine is a potent antimuscarinic, produces dry mouth and constipation. It has few extrapyrimidal side efeects: causes weight gain and carries a higher risk of worsening diabetes. Incidence of stroke may be increased in the elderly. Olanzapine is metabolized by CYP1A2 and glucuronyltransferase. The $\mathrm{t}_{1 / 2}$ is $24-30$ hours. Quetiapine is a new short acting atypical antipsychotic requires twice daily dosing. It blocks $\mathrm{D}_{2}, 5-\mathrm{HT}_{1}$ and $\mathrm{H}_{1}$ receptors in the brain. It had minimal extrapyrimidal side effects. However, it is quiet sedating and postural hypotension can occur. Weight gain and rise in blood sugar are infrequent. It is metabolized mainly by CYP3A4. The $t_{1 / 2}$ is 6 hours.

\section{Methodology and Results}

\section{Alcohol dependence and Reward Pathway in brain: A study to show the effect on brain Dopamine, Dihydrophenylacetic acid (DOPAC) and Homovanilic acid (HVA) in Rats}

Drugs that act as reinforcers indirectly activate a common biological mechanism, resulting in psychomotor stimulation. The mesolimbic dopamine system, with projection from the ventral tegmental area (VTA) of the midbrain to nucleus accumbens, appears to be particular importance in the mediation for drug reinforcement. At low and intermediate doses, ethanol stimulates dopamine synthesis, metabolism and turnover, and increases the firing rate of dopamine neurons in VTA. High Performance Liquid Chromatography (HPLC) studies have found that, ethanol produces an increase in dopamine release in nucleus accumbens. Conversely, ethanol with-drawal has been reported to decrease of extracellular dopamine levels. Male/Female Wistar rats (100$200 \mathrm{~g}$ ) maintained on ad libitium food and water was given daily 40 minutes access to $6 \%$ ethanol for 12-14 days and allocated to different treatment groups. Rats were sacrificed as per OECD guidelines and the excised brain homogenate was sent for HPLC to study the effect of Quetiapine and Olanzapine on alcohol dependence.

Animals: Male/Female Wistar rats of Rattusnorvegicus species belonging to the age group of 90 120 days with average body weight 100-200 g were used as experimental animals. The animals were housed in an animal facility for at least 4 days prior to testing with food and water available ad libitium. Then animals were allocated to differrent treatment groups (control and test groups).

Drugs: The drugs used were Quetiapine (36 $\mathrm{mg} / \mathrm{kg})$ and Olanzapine $(1.8 \mathrm{mg} / \mathrm{kg})$ obtained as a gift sample from Mepromax Pharmaceuticals, Deharadun. Drugs were injected orally as solution or suspension in saline containing $0.6 \%$ Carboxymethly cellulose. Ethanol was diluted to a $6 \% \mathrm{v} / \mathrm{v}$ solution in saline and water.

Analysis of brain Dopamine level: Each day during active dark cycle rats were given daily 40 minutes access to $6 \%$ alcohol solution and water for 12-14 days. Then control group received saline and the test group received test drug orally i.e. Quetiapine (36 mg/kg) and Olanzapine (1.8 $\mathrm{mg} / \mathrm{kg}$ ). Rats were sacrificed as per OECD guidelines and the excised brain was homogenized on ice in $5 \mathrm{ml}$ of $30 \%$ potassium hydroxide. Then the homogenate was kept in boiling water bath for 15 minutes. Then cooled on ice and added $0.2 \mathrm{ml}$ of saturated sodium sulphate solution, mixed thoroughly and kept on ice for 5 minutes. Centrifuged at $2000 \mathrm{rpm}$ for 10 minutes. Supernatent was collected and again recentrifuged 3-4 times. Supernatent was collected and sent for HPLC analysis. The HPLC system consisted of autosampler, CA-80 column and electrochemical detector. The mobile phase taken was $6.9 \mathrm{~g} /$ liter sodium monobasic phosphate, $250 \mathrm{mg} / \mathrm{liter}$ heptane sulphonic acid, $80 \mathrm{mg} /$ liter disodium EDTA and $50 \mathrm{ml} / \mathrm{liter}$ methanol. Buffer was adjusted to $\mathrm{pH} 3.6$ with HCL and pumped at the rate of $1.2 \mathrm{ml} / \mathrm{min}$. 


\section{JMSCR Vol||05||Issue||04||Page 20185-20192||April}

Statistical Analysis: Data were analyzed using one-way analysis of variance (ANOVA) followed by Dunnet's t-test.

Table: Effect of alcohol dependence on Dopamine levels in rats using HPLC

\begin{tabular}{|l|c|c|c|c|c|}
\hline Group No. & Treatment & Peak area & Peak height & Retention time & Total \\
\hline 1 & Control & 54.62 & 6.01 & 3.250 & 66.1 \\
\hline 2 & Alcohol treated & 190.69 & 19.59 & 3.250 & 211.0 \\
\hline 3 & Olanzapine treated & 94.94 & 10.08 & 3.250 & 95.59 \\
\hline 4 & Quetiapine treated & 147.9 & 15.59 & 3.250 & 154.26 \\
\hline
\end{tabular}

Note: The levels of dopamine in rat brain corresponds to peak area of the peak obtained in the graph.

\section{HPLC Graphs}

Fig 1: HPLC analysis for Alcohol group rats

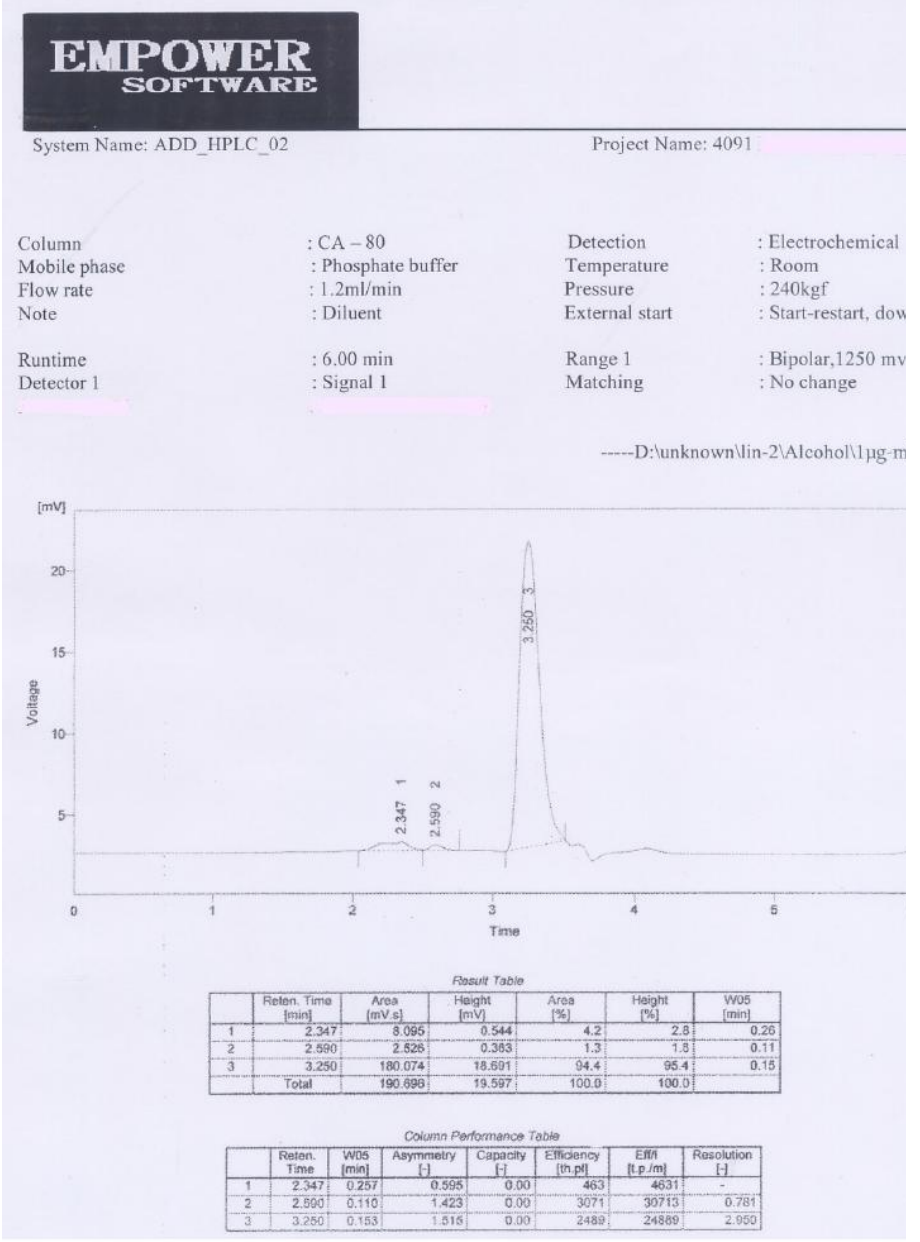

Fig 2: HPLC analysis for Control group rats

\section{EMPOWIRR
SOFTARE}

\begin{tabular}{llll}
\hline System Name: ADD_HPLC_02 & & Project Name: 4091 \\
& & & \\
& & & \\
Column & $:$ CA -80 & Detection & $:$ Electrochemical \\
Mobile phase & $:$ Phosphate buffer & Temperature & $:$ Room \\
Flow rate & $: 1.2 \mathrm{ml} / \mathrm{min}$ & Pressure & $: 240 \mathrm{~kg}$ \\
Note & $:$ Diluent & External start & $:$ Start-restart, down \\
Runtime & $: 6.00 \mathrm{~min}$ & Range 1 & $:$ Bipolar, $1250 \mathrm{mv}$ \\
Detector 1 & $:$ Signal 1 & Matching & $:$ No change
\end{tabular}

..-.-Dilunknownllin-1 lcontrolliug-m! 
Fig 3: HPLC analysis for Olanzapine group rats

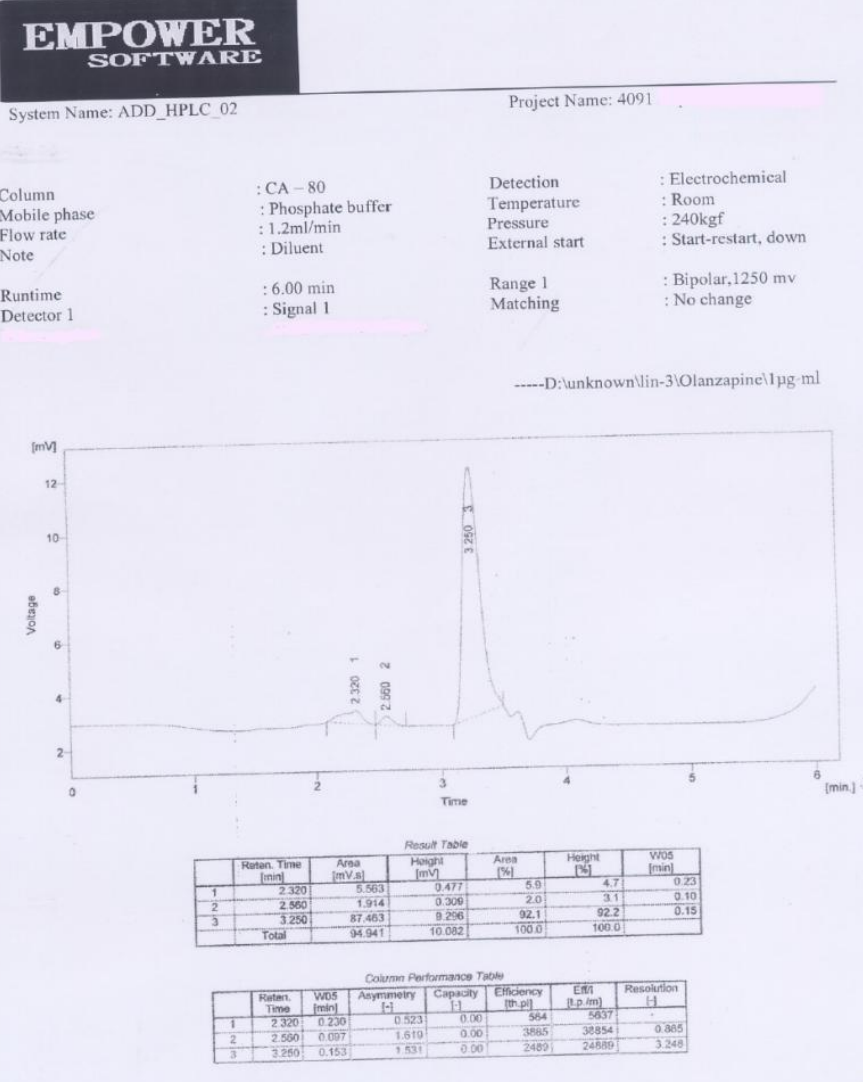

Fig 4: HPLC analysis for Quetiapine group rats

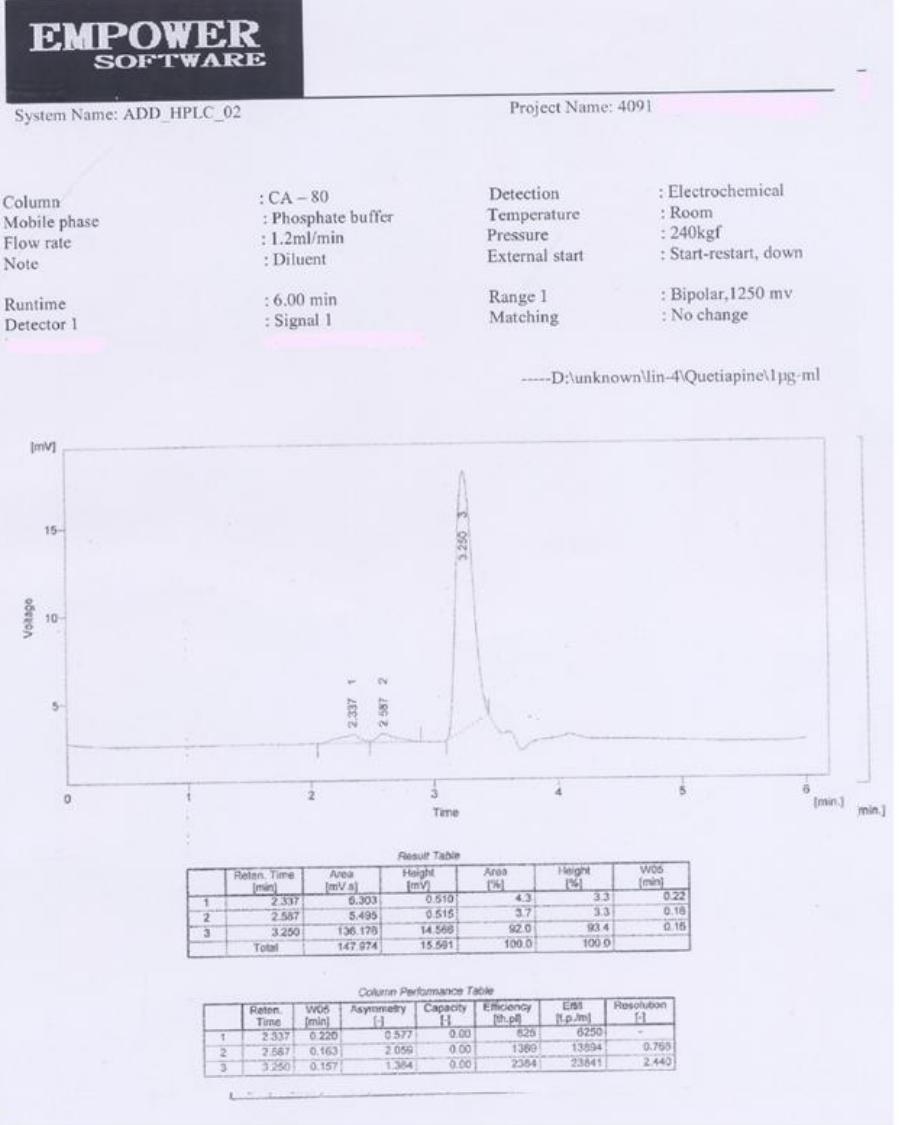

Drugs that act as reinforcers indirectly activate a common biological mechanism, resulting in psychootor stimulation. The mesolimbic dopamine system, with projection from the ventral tegmental area (VTA) of the midbrain to nucleus accumbens, appears to be particular importance in the mediation for drug reinforcement. At low and intermediate doses, ethanol stimulates dopamine synthesis, metabolism and turnover, and increases the firing rate of dopamine neurons in VTA. High Performance Liquid Chromatography (HPLC) studies have found that, ethanol produces an increase in dopamine release in nucleus accumbens. Conversely, ethanol withdrawal has been reported to decrease of extracellular dopamine levels. To measure the dopamine content in brain homogenate of rats were sacrificed as per OECD guidelines and HPLC analysis of the homogenate was done. The results of the test group were compared with the alcohol group. The peak area of control and alcohol group were found to be $54.62 \mathrm{mV}$.s and $190.69 \mathrm{mV}$.s respectively whereas the peak area of olanzapine and quetiapine group were found to be $94.94 \mathrm{mV}$.s and $147.9 \mathrm{mV}$.s respectively. The decreased in the peak area of the test group as compared to alcohol group shows that alcohol stimulated increased dopamine release was blocked by dopamine antagonists.

\section{Conclusions}

Chronic alcohol consumption profoundly affects the function of several vital organs particularly the liver, the skeletal muscle, the nervous system, gastrointestinal, cardiovascular and immune system. Chronic consumption of large amount of alcohol is associated with an increased risk of death. Death linked to alcohol consumption is caused by liver disease, cancer, accidents and suicide.

Alcohol dependence is common in $4-5 \%$ of the population and as with smoking difficult to treat effectively. Following detoxification, psychologycal therapy either in intensive inpatient or in outpatient rehabilitation programs serves as the primary treatment for alcohol dependence. Since 
these programs have been only moderately successful, with about $50 \%$ of patients relapsing within first year, there is much interest in finding drugs that can be useful adjuncts to psychological counseling. The first approach to pharmacotherapy was to deter drinking with drugs that cause a noxious reaction to alcohol by blocking its metabolism. The main pharmacological approaches are as follows:

- To alleviate the acute abstinence syndrome during drying out-benzodiazepines are effective, clonidine and propranolol are useful.

- To render alcohol consumption unpleasant- disulfiram is used.

- To reduce alcohol induced rewardnaltrexone is effective.

- To reduce craving- acamprosate is used.

The combination of Naltrexone and disulfiram should be avoided since both drugs are potential hepatotoxins. Compliance with disulfiram therapy is low and the evidence from clinical trials for its effectiveness is weak. It is no longer commonly used. The safety of disulfiram has not been demonstrated for the use in pregnancy. Naltrexone is contraindicated in hepatitis or liver failure. Considerable evidence from animal studies indicates that serotonergic and dopaminergic systems participate in the regulation of alcohol consumption. 3 In contrast, drugs that block dopamine receptors generally produce bad feeling i.e. dysphoric effects. Neither animals nor human beings will take such drugs spontaneously.4 A wide range of pharmacotherapy is available for the treatment of alcohol dependence and withdrawal have been introduced but none of them have shown proven efficacy and safety. Hence there is a new pharmacotherapy which is quite effective and safe. Dopaminergic antagonist, least likely to lower seizure threshold like Quetiapine and Olanzapine were selected. Dopaminergic antagonist, like Olanzapine and Quetiapine can be used as pharmacotherapy for alcohol dependence. Thus, this approach may provide a good pharmacotherapy for alcohol dependence and alcohol withdrawal.

\section{Acknowledgements}

The authors are grateful to Nitte University, Mangalore, for providing the necessary facilities to carry out this study.

\section{Articles related to dopamine and reward pathway}

$\checkmark$ Rueben A. et al., demonstrated that the neurobiological processes by which ethanol seeking and consumption are established and maintained are thought to involve areas of the brain that mediate motivated behavior, such as the mesolimbic dopa-mine system. Behavioral experiments consistently show that pharmacological manipulations of the dopamine transmission in the nucleus accumbens alter responding for ethanol, although ethanol reinforcement is maintained after lesions of the accumbal dopamine system. Additionally, extracellular dopamine increases in the nucleus accumbens during operant selfadministration of ethanol, which is consistent with a role of dopamine in ethanol reinforcement.

$\checkmark$ Shinshu Nakajima et al., reported that, the two subtypes of dopamine receptors, D1 and D2, play distinct roles in producing reinforcing effects. Experiments using SCH 23390 suggest that the reinforcing effects of food, water, saccharin, heroin and brain stimulation all critically depend on the activation of D1 dopamine receptors, particularly those in the nucleus accumbens and the ventral tegmental area. Raclopride, a D2 dopamine antagonist, also reduced barpressing responses for food, heroin, and brain stimulation. Thus, both D1 and D2 receptors seem to be involved in the reinforcement mechanisms. 
Richard J. Beninger et al., suggested that changes in overall level of activity of dopamine neurons appear to produce parallel changes in locomotor activity. Additionally, dopamine neurons seem to mediate in part the effects of biologically significant reinforcing stimuli on learning. It has been also suggested that dopamine hyper functioning underlies the development of schizophrenia.

\section{Articles related to alcohol dependence}

Robert et al., reported the effects of inhibiting dopamine $\mathrm{D}_{1^{-}}$or $\mathrm{D}_{2}$-like receptors in the ventral pallidum imposes a limit onethanol intake and extracellular levels of dopamine in the alcoholpreferringrat. $\mathrm{D}_{1}$ like antagonist, $\mathrm{SCH}-$ 23390 tended to decrease ethanol intake.

$\checkmark$ Dyr W et al., reported the effects of $D_{1}$ and $\mathrm{D}_{2}$ dopamine receptor agents on ethanol consumption in the high-alcoholdrinking (HAD) line of rats and showed that $\mathrm{D}_{1}$ antagonist SCH 23390 decreased ethanol drinking.

\section{Articles related to drugs olanzapine and} quetiapine

$\checkmark$ Eduard Vieta, Joseph $\mathbf{F}$ et al., reported evidence from randomized clinical trials regarding the effective dose range and optimal dose of quetiapine when treating bipolar I disorder patients with acute mania and also statistically significant improvement doses within the range of 400-800 mg/day. Dose escalation was rapid, with $92 \%$ of patients treated with monotherapy.

$\checkmark$ Per ThyrumT et al., reported that, atypical antipsychotic quetiapine provides equivalent efficacy to the typical antipsychotics chlorpromazine and haloperidol in the short-term treatment of schizophrenia. Moreover, the incidence of extrapyramidal symptoms associated with quetiapine treatment is equivalent to that observed with placebo treatment, which may lead to increased patient compliance with quetiapine compared with typical antipsychotics.

$\checkmark$ Pitschel-Walz, D. Abraham et al., reported that efficacy and tolerability of the new antipsychotics risperidone, olanzap-ine, sertindole and quetiapine in schizophrenia compared to placebo and conventional antipsychotics. All of the four new drugs are more effective than placebo, but the magnitude of the effect is only moderate. The drugs sertindole and quetiapine are as effective as haloperidol, and risperidone and olanzapine are slightly more effective than haloperidol in the treatment of schizophrenic symptomtology. All new antipsychotics are associated with less frequent use of antiparkinson medication than haloperidol. 